\title{
Reconfigurable Computing: Viable Applications and Trends
}

\author{
Alexandro M. S. Adário, Sergio Bampi \\ \{adario, bampi\}@inf.ufrgs.br \\ Instituto for Informatics - Federal University at Porto Alegre, Brazil \\ Av. Bento Gonçalves, 9500 - Porto Alegre(RS) Cx. Postal 15064 - CEP: $91501-970$
}

Key words: Reconfigurable architecture, image processing, FPGA

\begin{abstract}
The field of reconfigurable computing has experimented with different architectural and execution models in the last decade. As a radically new architecture approach, reconfiguration has been hindered by a lack of a common development framework and even an unified taxonomy to define the dynamics of the reconfiguration of the hardware. This paper reviews the taxonomy for the field and the previous experiments, which have clearly demonstrated the efficiency of the reconfiguration when, compared with general-purpose processors. The performance and power saving gains possible with dynamic reconfiguration are very high when compared to statically configured architectures. An experiment done with image processors with and without datapath reconfiguration is described, revealing the high area saving achievable in the case of neighborhood processors. The lack of clearly competitive and highly persive applications for the reconfigurable processors has hindered the necessary rethinking of the basic FPGA matrix architectures, as well as the development of a software framework for supporting the reconfiguration paradigm over a wider range of algorithms and applications.
\end{abstract}

\section{INTRODUCTION}

In the early 60 s, Gerald Estrin proposed the preliminary ideas of the field, which came to be known today as reconfigurable computing [Est63]. Estrin designed a "variable" system, consisting of 3 elements: a general-purpose 
computer, a variable hardware and a supervisory control unit. The variable hardware in this system allows the implementation of an arbitrary digital function, in a similar way a FPGA (Field-Programmable Gate Array) [Bro92][Fie94]can perform today. The concepts put forward by Estrin were far ahead of the implementation technology available at that time.

Until recently, reconfigurable architectures implemented in userconfigurable devices were not feasible, but with increasing levels of FPGA integration (above $200 \mathrm{~K}$ usable equivalent gates), as well as RISC cores and RAM merging into the reconfigurable arrays, the feasibility picture for such systems has changed dramatically.

This work presents a general overview of the advances in reconfigurable computing. An outline of the main differences between programmable and configurable circuits is initially provided. Dehon sets a useful definition [Deh96], always emphasizing that there are not clear boundaries between the sets of programmable and reconfigurable devices, but it is possible to distinguish the cases at the extremes:

a) Programmable - this term refers to architectures which heavily and rapidly reuse a single functional unit for many different functions. The canonical example is a conventional processor, which may perform a different instruction on its ALU every cycle.

b) Configurable - in the configurable devices, the active circuitry can perform any of a number of different functions, but the function can be or is changed in successive execution cycles. Once the device is configured to implement a function, it does not change during an operational period. The FPGA devices are the canonical example.

The following section of this paper discusses the motivation for the reconfiguration paradigm, followed by section 3 , which gives an account of the possible application areas to explore. Section 4 reviews previous relevant developments and experiments in reconfigurable computing, while section 5 and 6 set the taxonomy applicable to the hardware execution model and the programmability classes, respectively. Finally in section 7 an experiment comparing the application of hardware reconfiguration principles to a previously designed architecture for image processing is presented, showing the potential of this hardware design paradigm.

\section{WHY RECONFIGURABLE ARCHITECTURES?}

Advanced RISC microprocessors can solve complex computing tasks through a programming paradigm, based on fixed hardware resources. For most computing tasks it is cheaper and faster to develop a program in general-purpose processors (GPPs) specifically to solve them. While GPPs 
are designed with this aim, focusing on performance and general functionality, total costs of designing and fabricating RISC GPPs are increasing fast. These costs involve three parts:

a) Hardware costs: GPPs are larger and more complex than necessary for the execution of a specific task.

b) Design costs: functional units that may be rarely used in a given application may be present in GPPs, and may consume substantial part of the design effort. Considerable effort is given to increment the potential instruction level parallelism through superscalar control, so that the design effort is increasingly complicated by the demand for performance in the execution of potential application codes which are not known in advance

c) Energy costs: too much power is spent with functional units or blocks not used during a large fraction of the processing time. Solutions like clock slow-down, multiple internal supply voltages, and power-down are implemented in power managers of GPPs.

For specific applications or demanding requirements in terms of power, speed or costs, one may rely on either dedicated processors or reused core processors, which may be well suited to the application or optimized for a given set of performance requirements. In the former case, only the necessary functional units highly optimized for a specific range of problems may be present, which will result in unsurpassed power and area efficiencies for the application-specific algorithm. Until recently, application-specific processors (ASPs) implemented in configurable devices were not feasible, but with increasing levels of FPGA integration, as well as RISC cores and RAM merging into the reconfigurable arrays, the feasibility picture for userconfigured ASPs looks excellent, open the opportunity to break away from the general purpose processor paradigm.

Coupling a single one or a set of configurable devices to a GPP makes possible the exploitation of reconfigurable architecture. This structure can also aggregate some local or global-shared memory. The dynamic reconfiguration of the hardware has become a competitive alternative in terms of performance against a GPP software implementation, and it offers significant time-to-market advantage over the conventional ASP approach, in which a full-custom processor or ASIC has to be developed from specification to layout and subsequent fabrication.

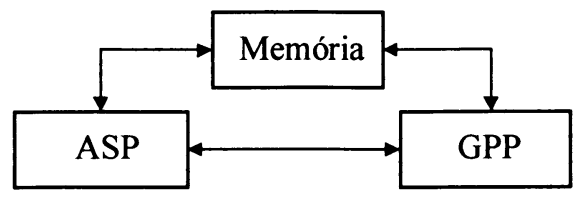

Figure 1. Generic Model of a Reconfigurable Architecture 
A reconfigurable architecture allows the designer to create new functions and to perform in customized hardware the operations that would take too many cycles to complete in a GPP. The GPP in Figure 1 may not include most of the complex functional units often designed-in, as well as considerable power and time can be saved. These units may be implemented on the configurable device, if and when demanded by the application.

\section{APPLICATION AREAS AND MARKET CONSIDERATIONS}

An important obstacle has hindered the commercial maturity of the reconfigurable hardware platform to date: no killer application demanding a large-volume production was found for this architectural model. A candidate application for a reconfigurable implementation must fulfill some basic characteristics: regularity, high concurrency due to data parallelism, small data operand size, and ability to execute in a pipeline organization with a simplified control.

Considering these characteristics, one may point out that some application which can have some advantage when using reconfigurability are: data encryption, decryption and compression; sequence and string matching; sorting; physical systems analysis and simulation; video and image processing; and specialized arithmetic.

A commercial system based on reconfigurable computing, which may include some of the applications mentioned above, can offer new features to the users and change the way the organizations solve their computing needs:

a) The system can aggregate the most recent trends imposed by consumers, because the design time is reduced and, in general, changes in design specification have less impact than on the traditional design approaches.

b) The savings in design/implementation time considerably reduce the time to market of entire systems.

c) The client support and assistance is easier, once the upgrades and bug fixing can be done remotely at a software level, just sending a new configuration.

d) The development process is more controllable and subject to parameterization, since the design cycle relies on software specification and software tools that follow the previously defined architectural model chosen.

e) Considering the end-user market, the number of interface boards in a conventional system can be reduced, once some applications are mutually exclusive or do not execute at the same time. 


\section{EXPERIMENTS ON RECONFIGURABLE COMPUTING}

Several reconfigurable architectures were designed in the last decade, showing the feasibility of this architectural approach: PAM [Ber89], SPLASH [Gok90], PRISM [Ath93], DISC [Wir95], Garp [Hau97] and NAPA1000 [Nat99].

PAM (Programmable Active Memories) is a project developed by DEC PRL and consists of an array of Xilinx FPGAs (25 XC3020 in the first version, Perle-0, and 24 XC3090 in the Perle-1[Vui96]). With dynamic reconfiguration, it has demonstrated the fastest implementation of RSA cryptography to that date [Sha93]. The set of application implemented using PAM also includes long integer arithmetic, computational biology, resolution of Laplace's equations, neural networks, video compression and image acquisition, analysis and classification.

SPLASH is a reconfigurable systolic array developed by Supercomputing Research Center in 1988. The basic computing engine of SPLASH is the Xilinx XC3090 FPGA, which composes each one of the 32 stages in the linear array. The second version of SPLASH, Splash 2, is a more generalpurpose reconfigurable processor array based on XC4010 FPGA modules. Each module is a processor element in the 16-module basic array boards. Splash2 can include until 16 array boards. The main application tested on the SPLASH architecture was related to computational biology.

PRISM is the acronym for Processor Reconfiguration through Instruction Set Metamorphosis and is a reconfigurable architecture for which specific tools have been developed such that, for each application, new processor instructions are synthesized. The tools for the PRISM environment use some concepts inherited from hardware/software codesign methods. The hardware/software partition process starts with a high-level specification using $\mathrm{C}$, and is user-guided. Two prototypes, PRISM-I and PRISM-II, have been built using Xilinx XC3090 and XC4010 FPGAs, respectively.

The Dynamic Instruction Set Computer, DISC, is a processor that loads complex application-specific instructions as required by a program. DISC uses a National Semiconductor CLAy FPGA and is divided in two parts: a global controller and a custom-instruction space. The instruction loading process is similar to the virtual memory mapping scheme: when a instruction is necessary, the global controller configures it and removes the unnecessary instructions if the custom-instruction space is full. Initially, a library of image processing instructions was created for DISC.

Garp is a reconfigurable architecture that sets a trend to incorporate RISC cores and FPGA arrays in the same die. It incorporates a MIPS-II instruction-set compatible core with a reconfigurable array that may 
implement co-processor functions as a slave computational unit located on the same die of the processor. Garp simulation results have shown a $24 \mathrm{X}$ speed-up over a software implementation in a UltraSparc 1/170 of the DES encryption algorithm. In an image dithering algorithm for a $640 \times 480$ pixels frame the speed up obtained by Garp was 9.4 times.

NAPA1000 is an architecture that integrates a 32-bit RISC processor (the NSC CompactRISC), a configurable logic structure, called ALP (Adaptive Logic Processor), memory and a ToggleBus network interface. The ALP is a $2 \mathrm{D}$ array of logic cells, dynamically and partially reconfigurable. The network interface allows the NAPA 1000 to be connected to 31 other NAPA1000. This architecture tried to combine three relevant architectural paradigms: scalar processing, reconfigurability and parallel computing. The prototyping and commercial introduction of NAPA was eventually discarded due to the killer-application effect.

\section{EXECUTION MODELS}

Page [Pag96] points out five design strategies by which programs may be embedded in reconfigurable architectures focusing on the relation between algorithm and implemented hardware: pure hardware, application-specific, sequential reuse, multiple simultaneous use and on-demand usage are the possible models for executing in hardware the computational task at hand.

In a pure hardware model, the algorithm is converted into a single hardware description, which is loaded into the FPGA. There is no relevant contribution of this model to reconfigurable architectures, since the configuration is fixed at design time and it never changes along the execution of the application. This model can be implemented using conventional HDLs and the currently available synthesis tools. The commercial maturity of FPGAs has thriven under this model, today widely practiced by hardware engineers.

PRISM is an example of an application-specific microprocessor (ASMP) execution model. In this system, the algorithm is compiled into two parts (Figure 2.a): an abstract machine code and an abstract processor. In the next step, the two are optimized to produce a description of an ASP and the machine code level algorithm implementation.

Very often an algorithm is too large to be implemented on the available devices or the design is area constrained by engineering or economic reasons. To overcome this constraint, the design is splitted into several parts, which are moved in and out of the devices, increasing the hardware density and producing a set of reconfiguration steps (Figure 2.b). This model of hardware execution of an algorithm execution is called sequential reuse, as 
only one of each Hw1, Hw2,..., Hwn configuration is present in the FPGA at any given time of the execution.

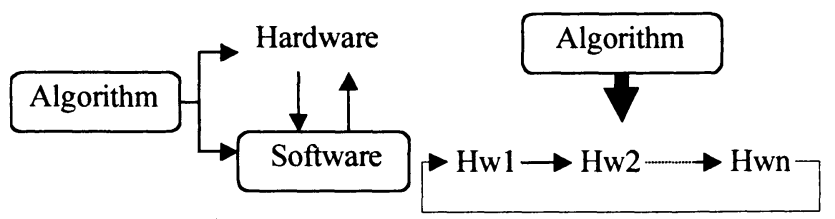

(a)

(b)

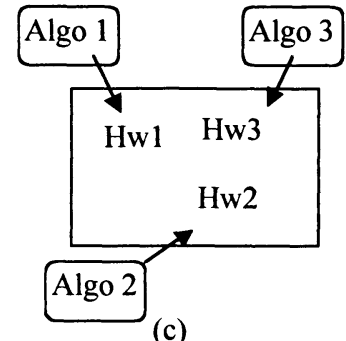

(c)

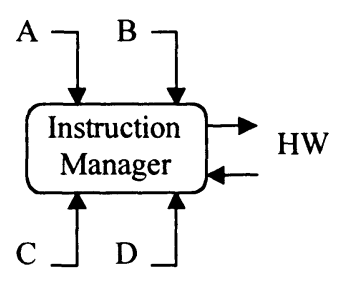

(d)

Figure 2. Example of Execution Models

If there is a large availability of configurable devices, many algorithms can be resident and execute simultaneously, interacting with various degrees of coupling (tightened or loose) with the host processor. The multiple simultaneous use model (Figure 2.c) is less common, requires more area than the sequential reuse, but is certainly very practical and amenable to the growing capacity of the FPGAs and the vastly different levels of demand for hardware of different algorithms. One may argue that most often than not it is the lack of memory capacity that prevents the full exploration of the FPGA resources for a given algorithm.

The last model, on demand usage (Figure 2.d), is very interesting for reconfigurable computing, and can be applied to a wide range of applications. This model is suitable for real-time systems and systems that have a large number of functions or operations, which are not used concurrently. The proposed dynamic instruction set computer (DISC) follows this strategy of configuring on-demand.

\section{PROGRAMMABILITY CLASSES}

The execution models presented by Page can be analyzed from the point of view of the reconfigurable architecture design. This classification divides the design models in programmability classes, considering the number of configurations and the time in which reconfiguration takes place: 
a) Static design (SD): The circuit has a single configuration, which is never changed neither before nor after system reset. The configurable device is fully programmed to perform only one functionality that remains unchanged during system lifetime. This class does not exploit the reconfiguration flexibility, taking advantage only of the implementation/prototipation facilities.

b) Statically reconfigurable design (SRD): The circuit has several configurations $(\mathrm{N})$ and the reconfiguration occurs only at the end of each processing task. This can be classified as run-time reconfiguration, depending on the granularity of the tasks performed between two successive reconfigurations. The configurable devices are better used and the circuit can be partitioned in this way, aiming for resources reusability. This class of architecture is called SRA (statically reconfigurable architecture).

c) Dynamically reconfigurable design (DRD): The circuit also has $\mathrm{N}$ configurations, but the reconfiguration takes place at runtime (RTR, RunTime Reconfiguration). This kind of design uses more efficiently the reconfigurable architectures. The timing overhead associated to this RTR procedure, during which no useful computation is done, has to be well characterized within the domain of the possible set of run-time configurations. The overall performance will be determined by the overhead-to-computing ratio. The implementation may use partially configurable devices or a set of conventional configurable devices (when one process, the others are reconfigured). The resultant architecture is called DRA (dynamically reconfigurable architecture).

SRD and DRD run-time reconfiguration advantages depend largely on the specific algorithm and its partition in sizable grain tasks. The reconfiguration overhead is heavily dependent on the FPGA microarchitecture, and it will be significantly decreased the convenient - yet costly - integration of FPGA + RISC core + SRAM within the same die. This set of on-chip functions provides for a fertile field of recent [Hau97] and upcoming innovations. The SRD hardware will certainly show better performance when compared to GPP software implementation, given the large time overhead incurred for reconfiguration in current commercial FPGAs. The DRD hardware will benefit the most from innovations in the fast reconfiguration arena, while requiring significant more effort in developing compiler optimization. The set of software support tools (runtime OS, profilers, compilers, loaders) necessary for this DRD represents considerable design effort. An effort already spent for the commercial GPP support, but not yet for the upbringing of the dynamically reconfigurable platforms. 


\section{A RECONFIGURABLE ARCHITECTURE FOR IMAGE PROCESSING}

DRIP (Dynamically Reconfigurable Image Processor) [Ada99] is a reconfigurable architecture based on the pipelined NP9 [Ada97], which is a static-design image processor based on the neighborhood processor architecture proposed by Leite [Lei94]. A neighborhood processor is a special computing device that simulates an array processor, a special class of parallel architecture consisting of simpler processors elements (PE)

The design goal for DRIP is to produce a digital image processing system using a dynamic reconfiguration approach, initially in a SRD scheme. Based on a previous NP9 design, done with conventional FPGAs in a static design scheme, we were expecting to obtain a considerable improvement in performance, with an operating frequency of $32 \mathrm{MHz}$ for real-time processing under a pipeline organization.

\subsection{The processor elements}

The PEs (Figure 3.a) of NP9 are functionally simple and can execute just two basic operations: addition and maximum. Each PE has two inputs (pixels X1 and X2), two weights (W1 and W2) associated to those inputs and one output $\mathrm{S}$. In the current model of the PE, weights can assume only three values: $-1,0$ and 1 . The DRIP/NP9 PEs are interconnected according to a data flow graph (Figure 3.b) which is based on a sorting algorithm..

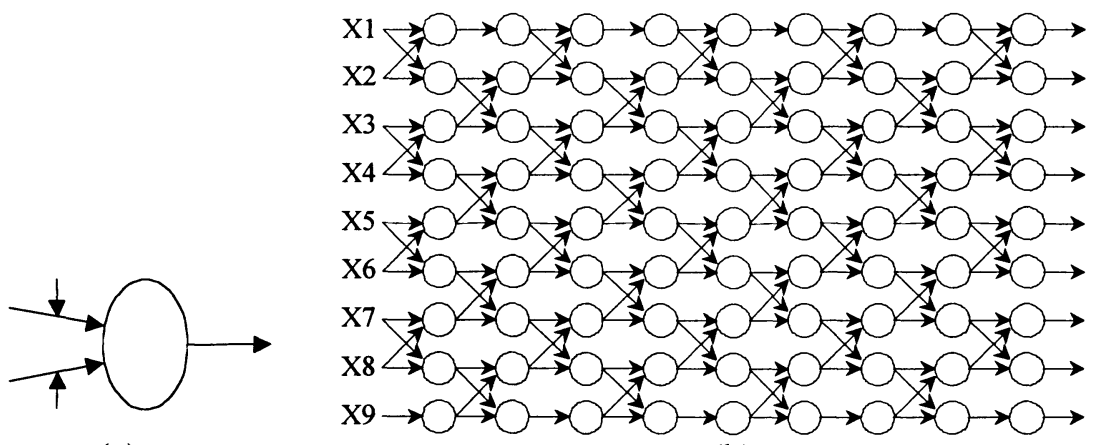

(a)

(b)

Figure 3. Model of the NP9/DRIP Processor Element(a) and Data Flow Graph (b)

DRIP architecture works with customized PEs. In the set of 18 possible PE functions, one may remove the redundant functions: those are equivalents or symmetrical on its inputs, thus reducing the effective number of functions 
to 8. Each highly optimized function is a component of a basic function library.

\subsection{Design Flow}

Typical design flow of the configuration of an algorithm onto DRIP is shown in Figure 4. First, an image processing algorithm is specified and simulated to verify its functionality. Later, the algorithm is optimized and translated to an intermediate representation, which matches the DRIP architecture.

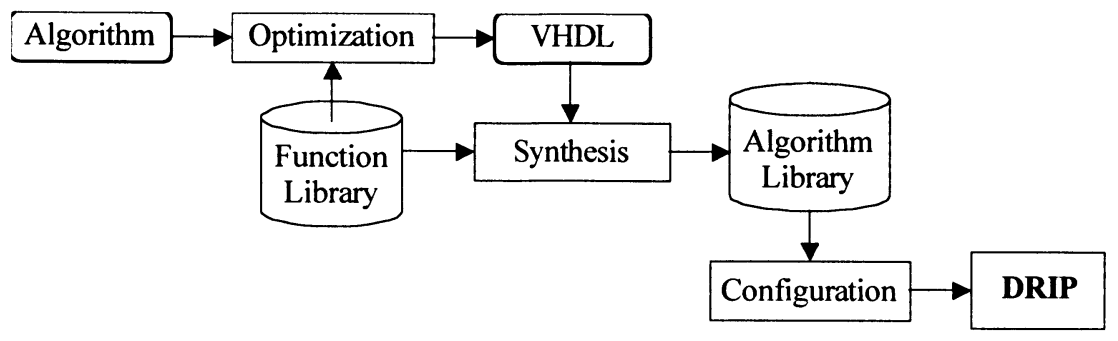

Figure 4. Design Flow of DRIP System

The synthesis process uses the previously designed function library, fully optimized for a better performance. The configuration bitstream that customizes the FPGA for the algorithm implementation is stored in a configuration library. The reuse of the modules of this library is essential for efficient implementation of several image processing functions. Once the configuration bitstream data is stored, it can be used repeatedly and over several modules of the entire architecture (Figure 5).

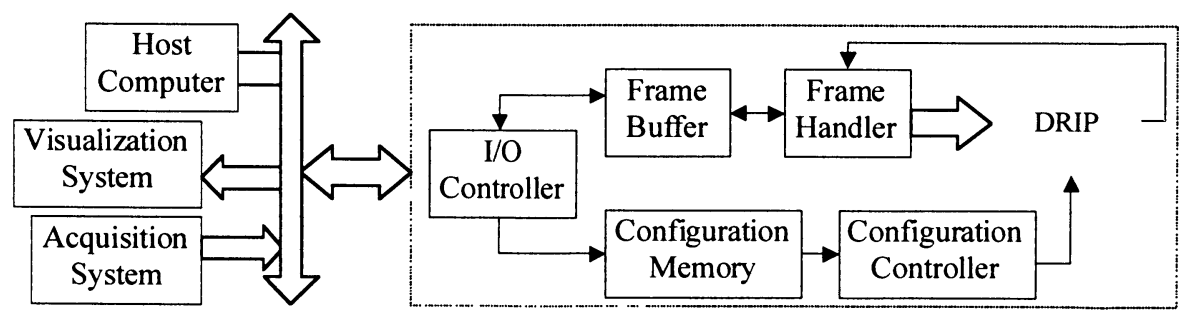

Figure 5. A Digital Image Processing System using DRIP

In the complete digital image processing system, DRIP is part of a more complex system, consisting of two memories (configuration memory and frame buffer, which stores a local copy of the image), the frame handler (which generates the neighborhood), and the configuration and I/O 
controllers. The most critical issue for the overall performance is bus bandwidth from the frame buffer to DRIP. The best solution is to design a single chip containing DRIP and the buffer handler, also including substantial sub-frame buffer memory.

\subsection{DRIP Results with Dynamic Reconfiguration}

The resource utilization in the NP9 design was inefficient, and the processor used 6,526 logic elements (LEs) in two FPGA devices (1 Altera Flex10K70 and 1 Flex10K100). DRIP achieved much better results for the same implementation technology, using only 1,113 LEs of a Flex10k30. This is $83 \%$ less area (for the worst-case configuration of DRIP) than the NP9 processor.

The target performance of the system is computed for real-time processing, considering 256 gray-level digital images of $1,024 \times 1,024$ pixels at a rate of 30 frames/s. The preliminary estimated performance of DRIP $(51.28 \mathrm{MHz})$ is $60 \%$ greater than the design target performance $(32 \mathrm{MHz})$ and almost $200 \%$ faster than the fixed hardware (non-reconfigurable) NP9 implementation (17.3 MHz).

\section{CONCLUSIONS}

There are plenty of experimental demonstration that the run-time hardware reconfiguration offers a significant performance advantage over the GPP software implementation. The main barriers for the commercial maturity of this technology were identified; on the practical side it is clear that the lack of a killer-application is an obstacle to the introduction of the significant developments that are needed in this field. In particular the pressing needs are: 1) the development of powerful software tools that support the mapping of high level language specifications into a runtime environment that automatically partitions the reconfigurable modules of the hardware. Co-synthesizers, compilers, linkers, and run-time support are complex if geared towards more general application codes; 2) on the hardware side, the development of FPGA families with fast reconfiguration capability, as the DPGA case, as well as considerable more memory megablocks to support a larger set of applications which demand both reconfiguration memory as well as large data sets. The image processing DRIP is a case in which the addition of considerable more memory benefits the application. The GPPs themselves are reserving increasing amounts of area to caching data and instructions closer to the decode/execute units; the shortcoming of currently commercial FPGAs is their lack of memory 
megablocks and of support for fast and/or partial reconfiguration. If FPGAs would incorporate those features, performance of DRD would then become even more appealing when compared to programmable GPP solutions.

\section{REFERENCES}

[Ada97] ADÁRIO, A. M. S.; CÔRTES, M. L.; LEITE, N. J. "A FPGA Implementation of a Neighbourhood Processor for Digital Image Applications" In: 10 Brazilian Symposium on Integrated Circuit Design, Gramado (RS), Brazil, Aug 1997 p. 125-134.

[Ada99] ADÁRIO, A. M. S.; ROEHE, E. L.; BAMPI; S. "Dynamically Reconfigurable Architecture for Image Processor Applications". In: 36 Design Automation Conference, New Orleans, June 1999. p 623-628.

[Ath93] ATHANAS, P.; SILVERMAN, H. F. "Processor Reconfiguration Through Instruction Set Metamorphosis". IEEE Computer, Mar 1993. p 11-18.

[Bro92] BROWN, Stephen. D.; FRANCIS, Robert J.; ROSE, Jonathan; VRANESIC, Zvonko G. Field-Programmable Gate Arrays. Kluwer Academic Publishers, 1992

[Ber89] BERTIN, P.; RONCIN, D.; VUILLEMIN, J.. Introduction to Programmable Active Memories. Paris: Digital Equipment Corporation, Paris Research Lab, June 1989. (PRL Report 3).

[Deh96] DEHON, A.. Reconfigurable Architectures for General-Purpose Computing. Massachusetts Institute of Technology, 1996. PhD Thesis. (AI Technical Report 1586)

[Deh97b]DEHON, A.. Role of Reconfigurable Computing. Berkeley: University of California. (http://www.reconfig.com/specrept/101596/session1/library/amdrc.htm (1997).

[Est63] ESTRIN, G.; BUSSELL, B.; TURN, R. "Parallel Processing in a Restructurable Computer System". IEEE Transactions on Electronic Computers, vol. EC-12, n. 5, Dec 1963. p 747-755.

[Fie94] FIELD-PROGRAMMABLE GATE ARRAY TECHNOLOGY. Boston: Kluwer Academic, 1994. Editor: TRIMBERGER, Stephen M.

[Gok90] GOKHALE, M. et al. "SPLASH: A Reconfigurable Linear Logic Array." In: International Conference on Parallel Processing, Aug, 1990. p 526-532

[Hau97] HAUSER, J. R.; WAWRZYNECK J. "Garp: A MIPS Processor with a Reconfigurable Coprocessor". In: IEEE Symposium on FPGAs for Custom Computing Machines, 1997. p 24-33.

[Lei94] LEITE, N. J.; BARROS, M. A.; "A Highly Reconfigurable Neighborhood Image Processor Based on Functional Programming". In: IEEE International Conference on Image Processing, Nov 1994. p 659-663.

[Nat99] NATIONAL SEMICONDUCTOR. NAPA1000. http://www.national.com/appinfo/ milaero/napa1000 (still available at Aug 1999).

[Pag96] PAGE Ian. Reconfigurable Processor Architectures. Microprocessors and Microsystems, May 1996. (Special Issue on Codesign).

[Sha93] SHAND, M.; VUILLEMIN, J. "Fast Implementations of RSA Cryptography". In: Symposium on Computer Arithmetic, 11., 1993, Los Alamitos, California. p. 252_259.

[Vui96] VUILLEMIN J. et al. "Programmable Active Memories: Reconfigurable Systems Come of Age". IEEE Transactions on VLSI Systems, Mar 1996.

[Wir95] WIRTHLIN, M. J.; HUTCHINGS, B. L. "A Dynamic Instruction Set Computer". In: IEEE Symposium on FPGAs for Custom Computing Machines, Apr. 1995. p 92-103 